\title{
A receptor-like kinase mutant with absent endodermal diffusion barrier displays selective nutrient homeostasis defects
}

\section{Alexandre Pfister ${ }^{1}$, Marie Barberon ${ }^{1}$, Julien Alassimone ${ }^{1 \dagger}$, Lothar Kalmbach ${ }^{1}$, Yuree Lee ${ }^{1 \ddagger}$, Joop EM Vermeer ${ }^{1}$, Misako Yamazaki ${ }^{1 \S}$, Guowei $\mathrm{Li}^{2}$, Christophe Maurel2 ${ }^{2}$ Junpei Takano ${ }^{3}$, Takehiro Kamiya ${ }^{4 \pi}$, David E Salt ${ }^{4}$, Daniele Roppolo ${ }^{1 \#,}$, Niko Geldner ${ }^{1 *}$}

'Department of Plant Molecular Biology, University of Lausanne, Lausanne, Switzerland; 'Biochimie et Physiologie Moléculaire des Plantes, CNRS/INRA/ SupAgro/Université Montpellier, Montpellier, France; ${ }^{3}$ Research Faculty of Agriculture, Hokkaido University, Hokkaido, Japan; ${ }^{4}$ Institute of Biological and Environmental Sciences, University of Aberdeen, Aberdeen, United Kingdom
*For correspondence: niko. geldner@unil.ch

Present address: ${ }^{\dagger}$ Department of Biology, Stanford University, Stanford, United States; ${ }^{\ddagger}$ Center for Plant Senescence and Life History, Institute for Basic Science, Daegu, Republic of Korea; Institute of Evolutionary Biology and Environmental Studies, University of Zürich, Zürich, Switzerland; "Graduate School of Agricultural and Life Sciences, University of Tokyo, Tokyo, Japan; "Institute of Plant Sciences, University of Bern, Bern, Switzerland

Competing interests: The authors declare that no competing interests exist.

Funding: See page 19

Received: 17 April 2014 Accepted: 21 August 2014 Published: 16 September 2014

Reviewing editor: Maria J Harrison, Boyce Thompson Institute for Plant Research, United States

cc) Copyright Pfister et al. This article is distributed under the terms of the Creative Commons Attribution License, which permits unrestricted use and redistribution provided that the original author and source are credited.

\begin{abstract}
The endodermis represents the main barrier to extracellular diffusion in plant roots, and it is central to current models of plant nutrient uptake. Despite this, little is known about the genes setting up this endodermal barrier. In this study, we report the identification and characterization of a strong barrier mutant, schengen 3 (sgn3). We observe a surprising ability of the mutant to maintain nutrient homeostasis, but demonstrate a major defect in maintaining sufficient levels of the macronutrient potassium. We show that SGN3/GASSHO1 is a receptor-like kinase that is necessary for localizing CASPARIAN STRIP DOMAIN PROTEINS (CASPs)—major players of endodermal differentiation-into an uninterrupted, ring-like domain. SGN3 appears to localize into a broader band, embedding growing CASP microdomains. The discovery of SGN3 strongly advances our ability to interrogate mechanisms of plant nutrient homeostasis and provides a novel actor for localized microdomain formation at the endodermal plasma membrane. DOI: 10.7554/eLife.03115.001
\end{abstract}

\section{Introduction}

The plant root is a highly selective filter that forages the soil environment for nutrients and water. Its function has been likened to that of an 'inverted gut', displaying a very similar dual role in uptake and protection (Waisel et al., 2002). Selectivity of roots is thought to crucially depend on the endodermis, an inner cell layer that surrounds the central vascular strand of the root and represents the main paracellular (apoplastic) transport barrier in young roots (Geldner, 2013). This apoplastic diffusion barrier is set up by the 'Casparian strips', lignin-like, hydrophobic impregnations of the primary cell wall that form a supracellular net-like structure around the central vasculature. In analogy to the tight junction of gut epithelia, this net-like arrangement provides a seal of the extracellular space, while still allowing for nutrient and water transport across outer and inner plasma membrane surfaces. The Casparian strip-bearing endodermis therefore represents an independent development of a polarized epithelium, evolved in the context of multi-cellular organisms with wall-bearing cells.

Later during endodermal development, hydrophobic (cork-like) suberin lamellae form all around the endodermal surface. This protective suberization should eventually abolish direct transport across the endodermal plasma membrane, forcing symplastic passage through plasmodesmata from outer cell layers. Despite being a central, conserved feature of higher plants, nothing was known until recently about the factors that build and position the Casparian strip. This led to the frustrating situation of not 
eLife digest Plant roots forage in the soil for minerals and water, but they must also provide a barrier that stops these nutrients leaking back out of the plant and stops microbes invading and causing disease. The endodermis-an inner layer of cells that surrounds the veins that run along the middle of a root-acts as such a barrier in young roots.

Polymers that repel water are deposited between the cells in the roots of almost all vascular plants - which include ferns, conifers, and flowering plants-to form a band around the endodermis called the 'Casparian strip'. This strip seals off the young roots and stops water moving through the gaps between plant cells, but still allows minerals, nutrients, and water to be transported through the root cells and into the plant. However, the importance of this structure has yet to be tested due to the lack of mutant plants without a Casparian strip.

Pfister et al. now report that deleting the gene that encodes a protein called SCHENGEN3 in the model plant Arabidopsis thaliana causes the Casparian strip to be interrupted by irregularly sized holes. This protein is normally found at high levels in the root endodermis, where it is embedded into the cell membranes. Pfister et al. also showed that without the SCHENGEN3 protein, other proteins called CASPs - that normally mark out a stripe around the root cells where the Casparian strip will form-only accumulated in discontinuous patches. Further experiments revealed that deleting the gene for SCHENGEN3 does not cause general problems in delivering the CASP proteins to the cell membrane; instead, it specifically stops the CASP proteins from forming a single, uninterrupted stripe.

Unexpectedly, disrupting the Casparian strip did not appear to hinder many of the functions of a root. The mutant plants could still take up water and nutrients, and the leaves of mutant plants had normal levels of many essential minerals-with the exception of potassium. The level of this mineral was much lower in mutant plants without the SCHENGEN3 protein. Pfister et al. suggest that in plants that lack an intact Casparian strip, potassium is continuously leaked from the root into the soil.

These findings reveal that in Arabidopsis, at least, the Casparian strip might not be as important as once thought for helping the plant to take up and accumulate water and nutrients. Further work is now needed to uncover the as yet unknown backup systems that might be able to compensate for the loss of this structure.

DOI: 10.7554/eLife.03115.002

being able to test the many supposed roles of the endodermis and its Casparian strip, because of an absence of specific mutants. Recent papers established that a family of previously undescribed fourtransmembrane-span proteins, called CASPs, forms a central, ring-like membrane domain in the endodermis, called the Casparian strip membrane domain (CSD). This domain acts as a lateral diffusion barrier and separates the endodermal plasma membrane into two distinct polar domains (Roppolo et al., 2011; Alassimone et al., 2010). Upon localization, the CASPs show extreme stability and a lack of endocytosis or lateral diffusion. Moreover, CASPs show extensive pair-wise interactions and associate strongly with cell walls. Multiple knock-outs of CASP family members display strongly disorganized formation of Casparian strips, demonstrating their functional importance. It is thought that the CASPs act as scaffolds that spatially organize cell wall biosynthetic enzymes. CASP1 was shown to determine the subcellular localization of a secreted peroxidase, PER64 (Lee et al., 2013). In current models, the role of CASPs is to assemble a specific NADPH oxidase with peroxidases, leading to oxidation of mono-lignols and local polymerization of lignin. Lignin formation in vivo must implicate numerous other cell wall proteins and ENHANCED SUBERIN 1 (ESB1), a dirigent-like protein is a cell wall protein that has also been demonstrated to be crucial for the correct formation of Casparian strips (Hosmani et al., 2013).

These recent breakthroughs held the promise that we might finally be able to investigate the functional relevance of the Casparian strip through analysis of the different mutants obtained. This however, turned out to be more difficult than expected. Multiple mutants of the CASP family display interrupted Casparian strips in the beginning, but rather quickly deposit more cell wall material in a delocalized fashion, eventually sealing the apoplast. A very similar phenotype occurs in the esb1 mutant (Hosmani et al., 2013). Moreover, both mutants display an earlier and stronger production of 
suberin lamellae (esb1 was named based on this phenomenon). This makes it difficult to use these mutants for assessing the importance of Casparian strips, since the partial defect on Casparian strip formation will always be confounded with the effect of ectopic/enhanced production of suberin in the same cells. In the rbohf mutant, the Casparian strip defect is only partial and restricted to the young part of the root. Moreover, RBOHF has roles in many different aspects of plant development and it would be hard to distinguish what phenotype is directly caused by defects in Casparian strip formation (Lee et al., 2013).

Here, we report the discovery of a novel Casparian strip mutant, schengen3 (sgn3). SGN3 encodes a receptor-like kinase with strong expression in the root endodermis. The SGN3 protein accumulates in the plasma membrane in a broad band within which the CSD forms. In its absence, only discontinuous patches of CASPs are observed. SGN3 loss-of-function leads to the strongest defects in Casparian strips known to date, with no indication of a compensatory upregulation of suberin, as seen for other mutants (Hosmani et al., 2013). Our analysis reveals a surprising capacity of the mutant to maintain homeostatic control in the absence of the major root diffusion barrier and challenges views according to which the root should lose its ability for selective nutrient uptake, because of a generalized, nonselective bypass of nutrients into the vasculature.

\section{Results}

\section{An endodermis-enriched receptor-like kinase is necessary for Casparian strip formation}

In a forward genetic, GUS-based screen for endodermal barrier mutants (Lee et al., 2013) (Alassimone et al., unpublished), we discovered one mutant, sgn3 that displayed a dramatic defect in endodermal barrier formation, as visualized by penetration of the apoplastic tracer propidium iodide (PI) into the stele along the entire length of the root. This resembled a phenotype of T-DNA insertion lines in an endodermis-enriched gene that we had analyzed in parallel (Figure 1A, Figure 1-figure supplement 1A) (Birnbaum et al., 2003; Brady et al., 2007). Complementation analysis and sequencing confirmed that the causal mutation in sgn3-2 was an early stop codon in the open reading frame of At4g20140. SGN3 encodes for a leucine-rich-repeat receptor-like kinase (LRR-RLK) of subfamily XI that had previously been described as GASSHO1 (GSO1) (Figure 1B, Figure 1-figure supplement 2, Figure 1-source data 1) (Tsuwamoto et al., 2008). In the previous publication, this LRR-RLK was investigated because of embryonic expression of a putative ortholog in Brassica napus, but no single mutant phenotype was found. A phenotype was only observed when gso1 (sgn3) was combined with a mutant of its closest homolog, At $5 \mathrm{~g} 44700$ (named GSO2). This gso 1 gso2 double mutant displayed a protodermal phenotype, characterized by a defective cuticle and fusion of cotyledons (Tsuwamoto et al., 2008). True leaves did not differ from wild type. In contrast to SGN3/GSO1, GSO2 was shown to be expressed only in shoots, but not in roots, explaining the exclusive root phenotype in the sgn3 single mutant that we report here and that had escaped detection earlier on. In order to investigate the cause of this phenotype, we investigated formation of the Casparian strip and found that it is still formed and correctly positioned, but that it is repeatedly interrupted, forming irregularly-sized 'holes' of several micrometer in length (Figure 1C, Figure 1-figure supplement 1B). Suberin lamellae formation, by contrast, appeared at a position and in a manner very similar to wild type (Figure 1D,E, Figure 1-figure supplement $1 C, D$ ).

\section{The Casparian strip membrane domain and lateral diffusion barrier is defective in the sgn3 receptor-like kinase mutant}

We asked whether the Casparian strip cell wall defect is caused by a defect in the formation of the CSD, or whether the function of SGN3 lies downstream of the CASPs, as has been demonstrated for the NADPH oxidase SGN4/RBOHF (Lee et al., 2013). In the sgn3 background, CASP1-GFP showed a pattern of interrupted patches that was strikingly similar to that of the Casparian strip itself. This suggests that it is the incorrect localization of the CASPs that causes the Casparian strip defects of the mutant (Figure 1F). Interestingly, in a novel forward genetic screen in which we scored directly for mutants with altered CASP1-GFP localization (Kalmbach et al., unpublished), we found 14 novel alleles of sgn3 (Figure 1B, Figure 1-figure supplement 2, Figure 1-source data 1). This suggests that SGN3 is one of the major non-redundant genes necessary for CASP localization in the Arabidopsis genome. We could show that not only CASP1-GFP, but all four other CASPs (CASP2-5) display identical defects in sgn3 (Figure 1-figure supplement 1E). 


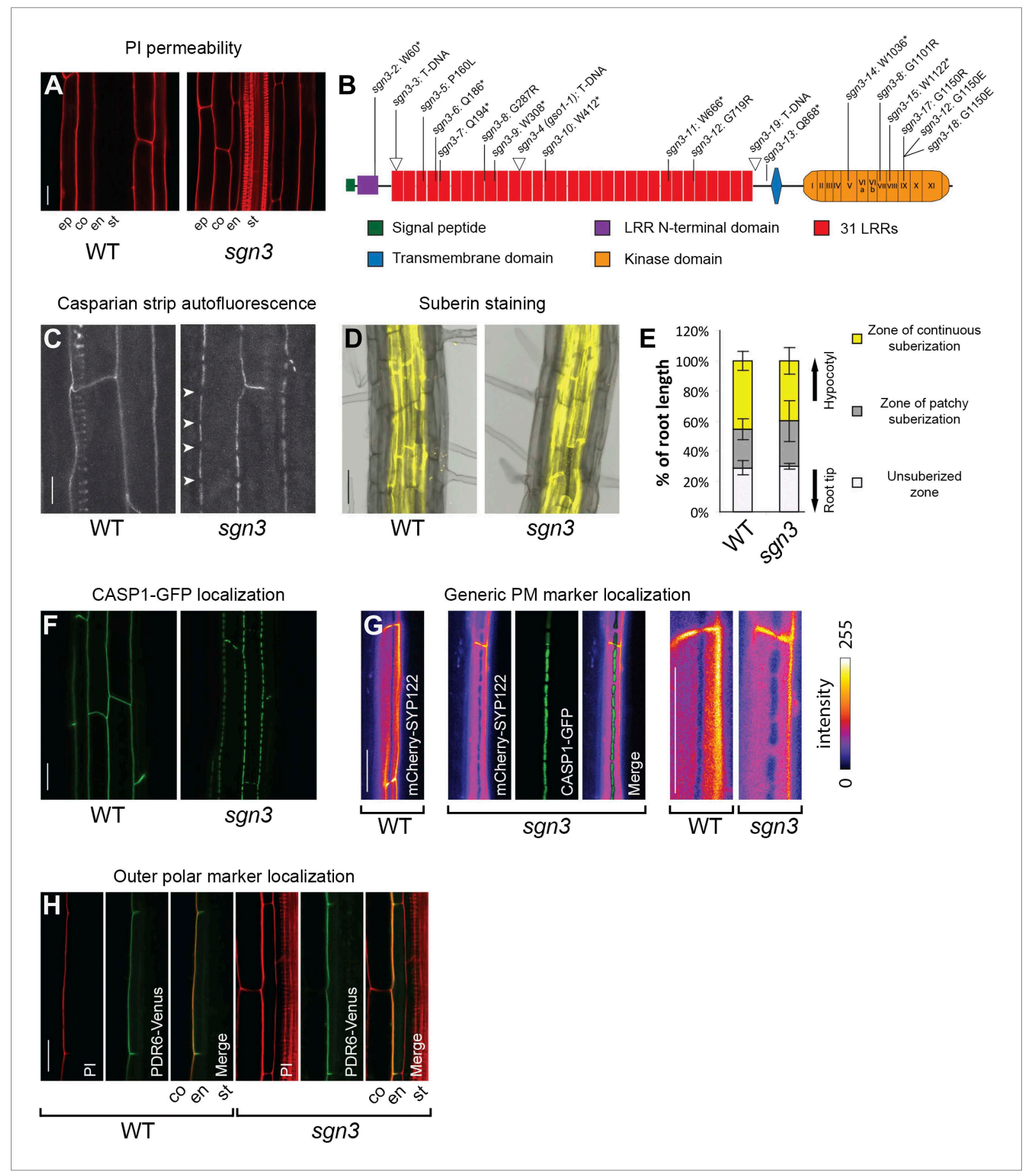

Figure 1. SGN3 receptor-like kinase is important to establish a functional endodermal barrier. (A) Lack of endodermal diffusion barrier in sgn3-3 visualized by presence of propidium iodide (PI) in stele. (B) Diagram of the SGN3 protein showing the different domains, T-DNA insertion lines (indicated with triangles) and the ethyl methanesulfonate (EMS)-induced mutations (see also Figure 1-figure supplement 2). (C) Surface view of Casparian strip, visualized by autofluorescence after clearing. Note discontinuous lignin deposition in sgn3-3. Pictures are maximum projections of confocal z-stacks. Arrowheads indicate discontinuities in sgn3. Spiral-like signal in WT is from deeper-lying xylem vessel. (D) Fluorol yellow staining of suberin lamellae deposition in sgn3-3 and WT. Pictures are overlays of transmitted light image (gray) with fluorescent signal from suberin dye (yellow). (E) Occurrence of suberin deposition along the root is not altered in sgn3-3. Suberin lamellae deposition was quantified considering three different zones: non-suberized zone, zone of patchy suberization, and zone of continuous suberization ( $n=5$, one representative experiment presented). (F) Surface view of CSD network visualized with CASP1-GFP expressed under CASP1 promoter showing the net-like structure with discontinuities in sgn3-3. Projections as in C. (G) Absence of a lateral diffusion barrier in sgn3-3 visualized with plasma membrane marker line CASP1::mCherry-SYP122 (intensity color coded). Figure 1. Continued on next page 
Figure 1. Continued

Confocal pictures were taken at the surface of an endodermal cell. Note the mutually exclusive localization with the CSD marker CASP1-GFP (green). Two right images are magnification of the two leftmost images. $(\mathbf{H})$ Localization of the outer marker PDR6-Venus expressed under CASP1 promoter is still polar in sgn3-3. Pictures are median longitudinal sections of endodermal cells. Scale bars: A, C, F, G, H = 20 m; $\mathbf{D}=50 \mu \mathrm{m}$. ep, epidermis; co, cortex; en, endodermis; st, stele; LRR, Leucine-rich repeat.

DOI: 10.7554/eLife.03115.003

The following source data and figure supplements are available for figure 1:

Source data 1. Detail of SGN3 T-DNA and EMS mutants.

DOI: 10.7554/eLife.03115.004

Figure supplement 1. Both Casparian strip domain and Casparian strip but not the suberin are affected in sgn3.

DOI: 10.7554/eLife.03115.005

Figure supplement 2. Diagram of SGN3 genomic DNA with T-DNA and EMS mutants.

DOI: 10.7554/eLife.03115.006

An interrupted CSD should abolish the lateral diffusion barrier in the plasma membrane of endodermal cells that we had previously described (Alassimone et al., 2010). Indeed, a generic plasma membrane protein that is excluded from the CSD in wild type (Alassimone et al., 2010) (Figure 1G) shows a pattern of exclusion in the mutant that represents a perfect negative image to the CASP1-GFP islands (Figure 1G). This illustrates that the lateral diffusion barrier between inner and outer plasma membrane domain is absent in sgn3 mutants, but that the remaining CSD islands are still able to exclude other proteins. To our surprise, this defect did not affect the polar localization of PDR6, a transporter that localizes to the outer polar domain in the endodermis (Figure 1H). This indicates that a strict polarity in the endodermis can be maintained in the absence of a diffusion barrier, further supporting the notion that polarity of transmembrane proteins in plants might simply be maintained by generally low lateral diffusion, high rates of endocytosis, or both (Geldner, 2009).

\section{CASP stability and cell wall attachment remain intact in the sgn3 mutant}

We had shown previously that during endodermal differentiation, CASP1-GFP changes from a protein that displays endocytic cycling and lateral diffusion to one that is highly stable and immobile in the CSD (Roppolo et al., 2011). In this process, CASP1-GFP localization passes through a 'string of pearls' stage, in which individual islands or 'patches' of CASP1-GFP are aligned in a band before eventually fusing (Figure 2A). Comparison of 3D-time-lapse observations of endodermal differentiation revealed that sgn3 is not able to progress from this 'string of pearls' stage, but only undergoes partial fusion of individual islands, leading to CASP1-GFP patches of heterogenous size (Figure 2A). We further found by fluorescence recovery after photobleaching (FRAP) that these CASP1-GFP islands have similar stability and turnover as wild type (Figure 2B). Another feature of the CSD is its very strong adherence to the cell wall, leading to the long-described phenomenon of 'band plasmolysis' in endodermal cells (Krömer, 1903; Behrisch, 1926; Alassimone et al., 2010). The inability of the CASP1-GFP ring to retract from the Casparian strip upon plasmolysis (Figure 2-figure supplement 1) allow for easy visualization of this phenomenon. This adhesion leads to greatly flattened, often fenestrated protoplasts, that looked very unlike the usual, rounded protoplasts of epidermal cells (Alassimone et al., 2010). sgn3 showed the very same inability of CASP1-GFP to retract from the strips, demonstrating that plasma membrane-to-cell wall attachment remains intact in the mutant (Figure 2-figure supplement 1). We conclude that sgn3 does not cause a general defect in CASP1-GFP trafficking, polymerization or cell wall attachment, but rather a specific defect in the progression of already stable CASP islands towards a contiguous band.

\section{SGN3 localizes to the plasma membrane and is expressed early during endodermal differentiation}

We then generated lines of tagged SGN3 variants in order to investigate its expression and subcellular localization. Only a C-terminal mVenus fusion with a 9.4-kb genomic fragment containing intron, 5'UTR, and the upstream neighboring gene, provided full complementation of the mutant phenotype, indicating functionality of the protein fusion and of its regulatory sequences (Figure 2-figure supplement 2A,B). 

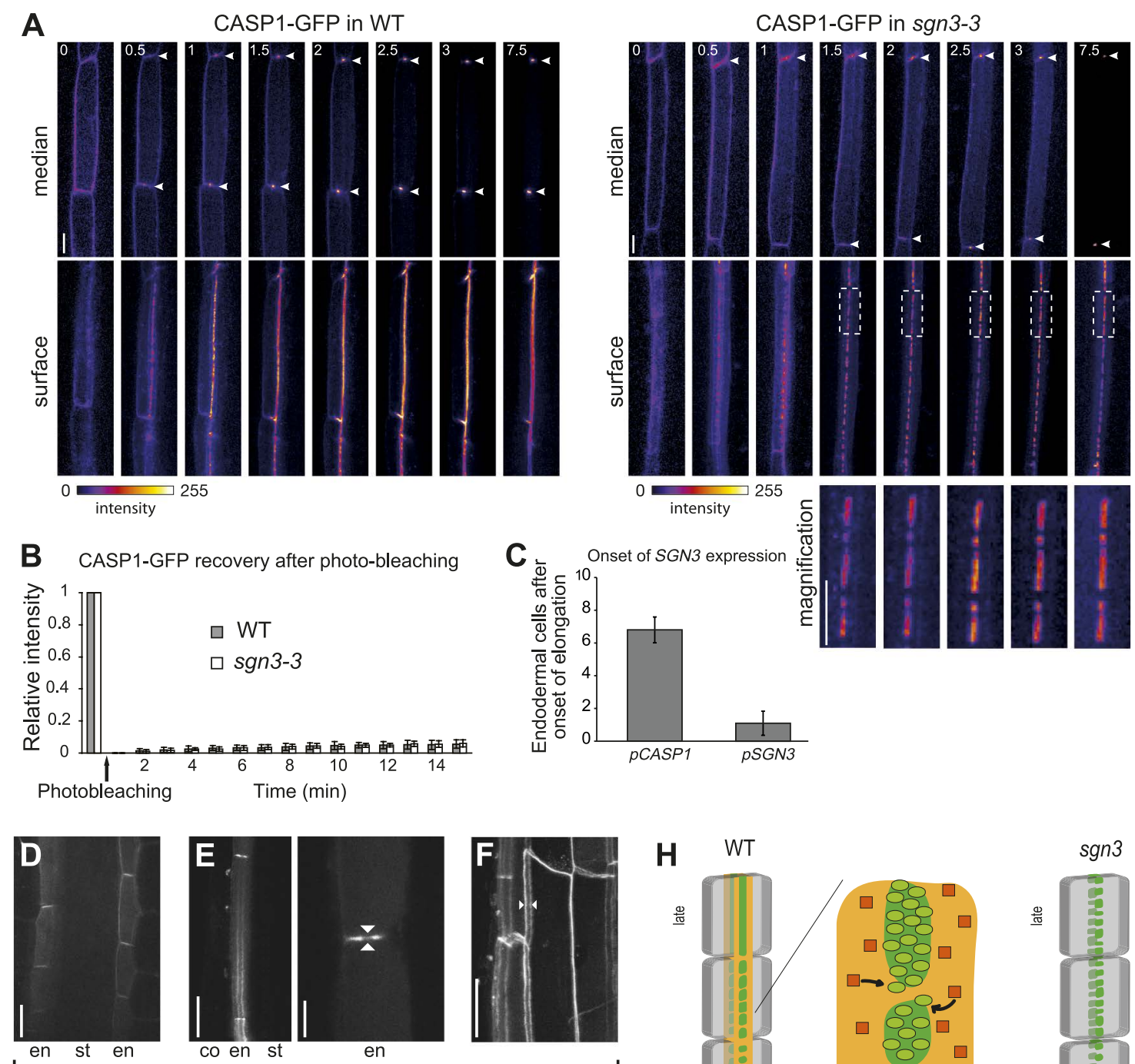

pSGN3::SGN3-mVenus

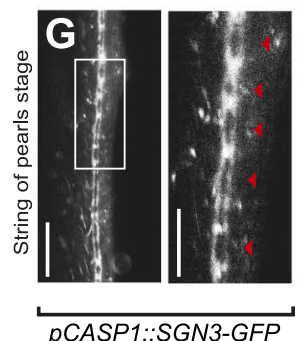

Casparian Strip Domain

CASP1

SGN3 Domain

SGN3

$\mathbf{H}$

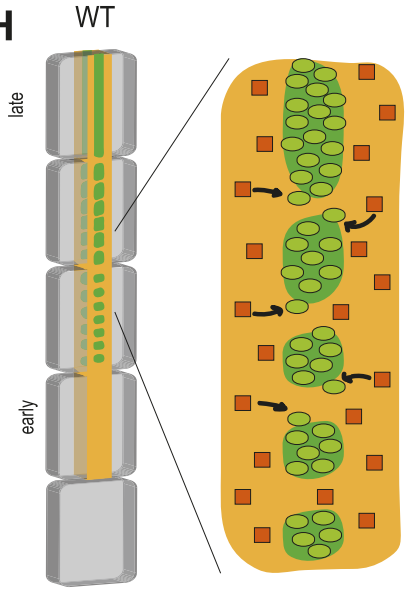

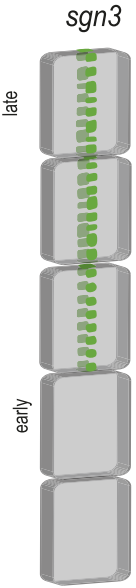

Figure 2. SGN3 localizes on both sides of the Casparian strip domain and is important for the CASP1-GFP patches to fuse into a contiguous band. (A) 3D-confocal time lapse imaging of CASP1::CASP1-GFP in Col-0 and sgn3-3 background reveals problems with progression of CASP1-GFP localization in sgn3-3. Images show median and surface image of endodermal cells of the same root. Arrowhead indicates CSD, dotted box in sgn3-3 shows immobility of CSD islands. Time in hours. (B) Fluorescence recovery after photobleaching (FRAP) with pCASP1::CASP1-GFP in WT (gray) and sgn3-3 (white). The fifth cell after the onset of CASP1 expression was used. Similar very low recoveries are observed in both genotypes after 15 min. $\mathrm{n}=8$ independent assays for each series, error bars = s.d. (C) SGN3 is expressed before the onset of CASP1 expression in the endodermis. Quantification was done using pSGN3::SGN3-mVenus and pCASP1::CASP1-GFP. The onset of expression corresponds to the first cell in the endodermis file with a clear fluorescent signal not visible in WT. $n=10$. Error bars $=$ s.d. (D) Localization of SGN3-mVenus under its own promoter in elongating endodermal cells. (E) In differentiated endodermal cells, SGN3-mVenus accumulates in the transversal and anticlinal sides of the plasma membrane, but is depleted from the CSD. Left panel shows a transition from a median (top) to a surface (bottom) view of an endodermal cell. Right panel shows a close-up of a median Figure 2. Continued on next page 
Figure 2. Continued

view with SGN3 surrounding the CSD. Arrowheads indicate the CSD. (F) Maximum projection of a z-stack showing the localization of SGN3 on both sides of the CSD. Note the CSD surrounded by two SGN3-mVenus 'lines' (white arrowheads). (G) Localization of SGN3-GFP under CASP1 promoter in the 'string of pearls' stage. SGN3 surrounds individual CSD patches preceding their fusion (left panel). Close-up showing the CSD patches (red arrowhead) surrounded by SGN3-GFP (right panel). (H) Schematic of SGN3 putative mode of action. The SGN3 kinase might promote addition of new CASP units to already formed CASP microdomains. Scale bars: A, G (left panel), $10 \mu \mathrm{m} ; \mathbf{D}, \mathbf{E}$ (left panel), F, $20 \mu \mathrm{m}$; $\mathbf{E}$ (right panel), $\mathbf{G}$ (right panel), $5 \mu \mathrm{m}$. Ep, epidermis; co, cortex; en, endodermis; st, stele.

DOI: 10.7554/eLife.03115.007

The following figure supplements are available for figure 2:

Figure supplement 1. Plasma membrane-to-cell wall attachment remains intact in sgn3 upon plasmolysis.

DOI: $10.7554 /$ eLife.03115.008

Figure supplement 2. SGN3 genomic construct and sgn3 PI phenotype complementation. DOI: 10.7554/eLife.03115.009

Figure supplement 3. Schematic illustrating quantification of onset of expression along the root. DOI: 10.7554/eLife.03115.010

In transgenic lines of this construct, expression of SGN3 could be observed in endodermal cells shortly after the onset of elongation, preceding the onset of CASP1 expression (Figure 2C, Figure 2-figure supplement 3). This is consistent with a function of SGN3 in establishing correct CASP1 localization. Initially, SGN3 could be found on all cell sides, but it then quickly accumulated in the transversal and anticlinal sides of the plasma membrane, yet became excluded from the CSD itself-a hitherto unknown subcellular localization pattern (Figure 2D-F). In 3D-reconstructions this leads to the appearance of a broader ring-like domain that flanks the more restricted, median CSD (Figure 2F). During the critical phase of CASP domain progression from isolated islands towards a fused ring-at which the sgn3 phenotype becomes apparent-SGN3 is seen to surround individual CASP islands on all sides (Figure 2G). Such localization would fit with a role of SGN3 in promoting fusion of growing CASP islands, such that an uninterrupted band can be formed (Figure $2 \mathrm{H}$ ).

\section{SGN3 is required for the enhanced suberin production of other Casparian strip-defective mutants}

In order to establish the functional relationships between SGN3 and other previously characterized genes, we combined sgn3 with casp 1 casp3 double and esb1 single mutants. ESB1 is an extracellular protein that localizes to the site of Casparian strip formation and whose absence causes a phenotype resembling that of sgn3 insofar as both the Casparian strip itself and CASP1-GFP are localized in interrupted bands (Hosmani et al., 2013). In contrast to sgn3, however, esb1 mutants additionally display increased, delocalized deposition of additional autofluorescent cell wall material as well as enhanced and earlier deposition of suberin (Hosmani et al., 2013). We found ESB1-mCherry in sgn3 to be both present and correctly localized, that is, specifically accumulating at the remaining CASP1-GFP islands (Figure 3A). Thus, ESB1 does not require SGN3 for its accumulation and localization and the Casparian strip discontinuities in esb1 and sgn 3 might be caused by independent mechanisms. Consistently, we found that sgn3 esb1 double mutant formed less and/or smaller Casparian strip patches than each single mutant, indicating additivity of the two mutations in this common aspect of their phenotypes (Figure 3B,C). Intriguingly however, sgn3 esb1 double mutants neither display an increased or delocalized deposition of autofluorescent cell wall material, nor an enhanced and earlier formation of suberin. This indicates a full epistasis of sgn3 over esb1 for this phenotype (Figure 3B,D). The same epistatic relationship was found between sgn3 and casp1 casp3 double mutants (Figure 3B-D). The enhanced and ectopic formation of autofluorescence and suberin is thought to be due to a feedback and crosstalk between cell wall components, connecting correct Casparian strip formation to the further deposition of lignin and suberin lamellae formation (Hosmani et al., 2013). The epistasis of sgn3 suggests that the perception/signaling that leads to enhanced autofluorescence and suberin formation in esb1 and casp 1 casp3 is mediated by-or at least requires-the SGN3 receptor-like kinase.

\section{sgn3 growth phenotypes are extremely sensitive to environmental conditions}

Considering the strength of the barrier phenotype of sgn3 and its inability to initiate potentially compensatory suberization, we were surprised to observe a rather mild growth phenotype of sgn3 under 
A

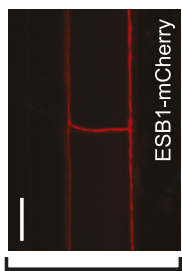

WT
ESB1 localization

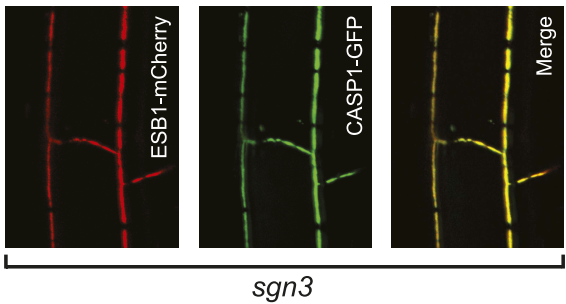

$\operatorname{sgn} 3$

B
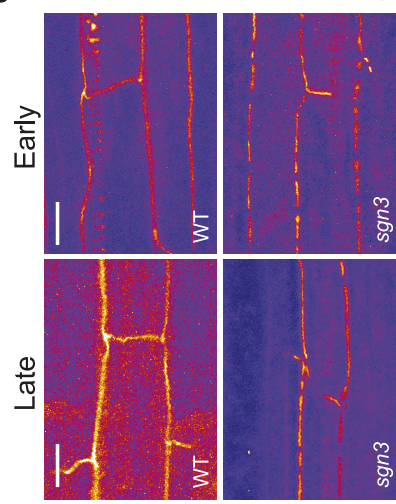

Casparian strip autofluorescence
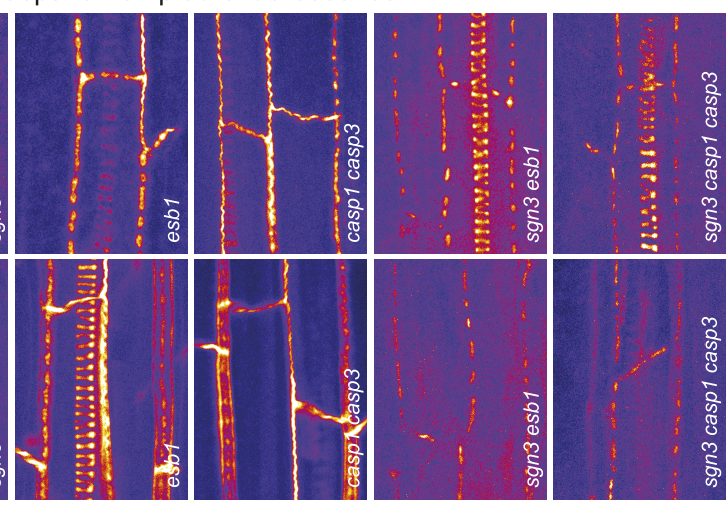

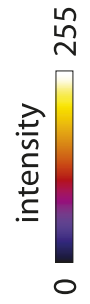

C

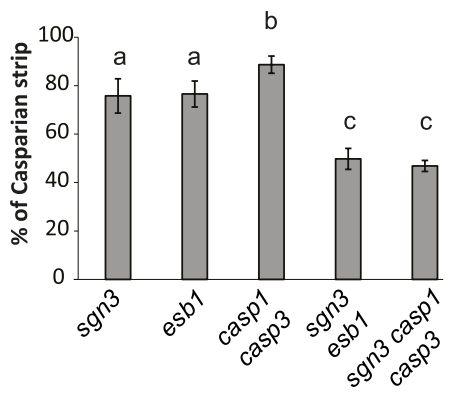

D $120 \%$

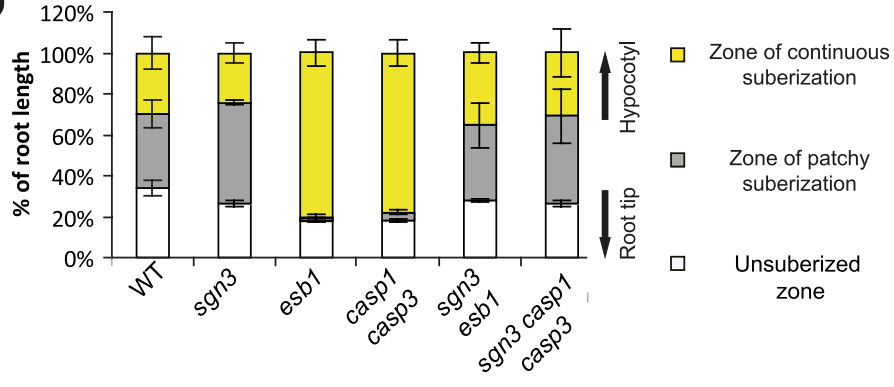

Figure 3. Relations between sgn3 and molecular players in Casparian strip formation. (A) ESB1-mCherry localizes to the CASP1-GFP microdomains in sgn3. ESB1-mCherry and CASP1-GFP are expressed under their own promoters. Pictures are maximum projections of confocal z-stacks. (B) Surface view of Casparian strip, visualized by autofluorescence after clearing. Top and bottom panels correspond to the early and late stages of endodermal differentiation, 15 and 30 cells after onset of elongation, respectively. When sgn 3 is crossed to casp 1 casp 3 or esb 1, note the additive effect of these mutants concerning the CS patches (quantified in C). Note also the absence of enhance/ectopic deposition of lignin in sgn3 esb1 and sgn3 casp1 casp3. Pictures are maximum projections as in A. Strong spiral-like signals are from protoxylem cells. (C) Quantification of Casparian strip presence shows an additive effect in sgn3 esb1 and sgn3 casp1 casp3. Casparian strips were quantified as the percentage of cell wall showing autofluorescence along a line of Casparian strip signal of a given length. $n=10$. (D) sgn3 is epistatic to esb1 and casp1 casp3 for ectopic suberin deposition. 5-day-old seedlings were stained with Fluorol Yellow. Quantification was done as in Figure 1E. Bars represent the percentage for each zone along the root ( $\mathrm{n}=5$, one representative experiment). $\mathbf{C}$, Error bars = s.d., different letters indicate significant differences between genotypes, determined by analysis of variance (ANOVA) and Tukey test as post hoc analyses $(p<0.05)$. Scale bars: A, B, $10 \mu \mathrm{m}$. sgn3 is sgn3-3, esb1 is esb1-1, casp1 is casp1-1 and casp3 is casp3-1.

DOI: 10.7554/eLife.03115.011

certain conditions (Figure 4A, Figure 4-figure supplement 1A). Yet we noticed that sgn3 growth is extremely sensitive to changes in environmental conditions that are non-stressful to wild type (Figure 4A, Figure 4-figure supplement 1A). We ensured that the endodermal barrier phenotype persisted in developed root systems of rosette stage plants both on soil and on hydroponics, thus excluding that the weak phenotype is caused by an eventual repair of the barrier in older plants (Figure 4-figure supplement 1B). A systematic variation of day length, temperature, and light-intensity revealed that growth differences to wild type are more pronounced at higher temperature, as well as under long-day conditions (Figure 4B, Figure 4-figure supplement 1C). In general, sensitivity of sgn3 to growth conditions is such that we observed everything from a near absence of phenotypes to severely dwarfed 


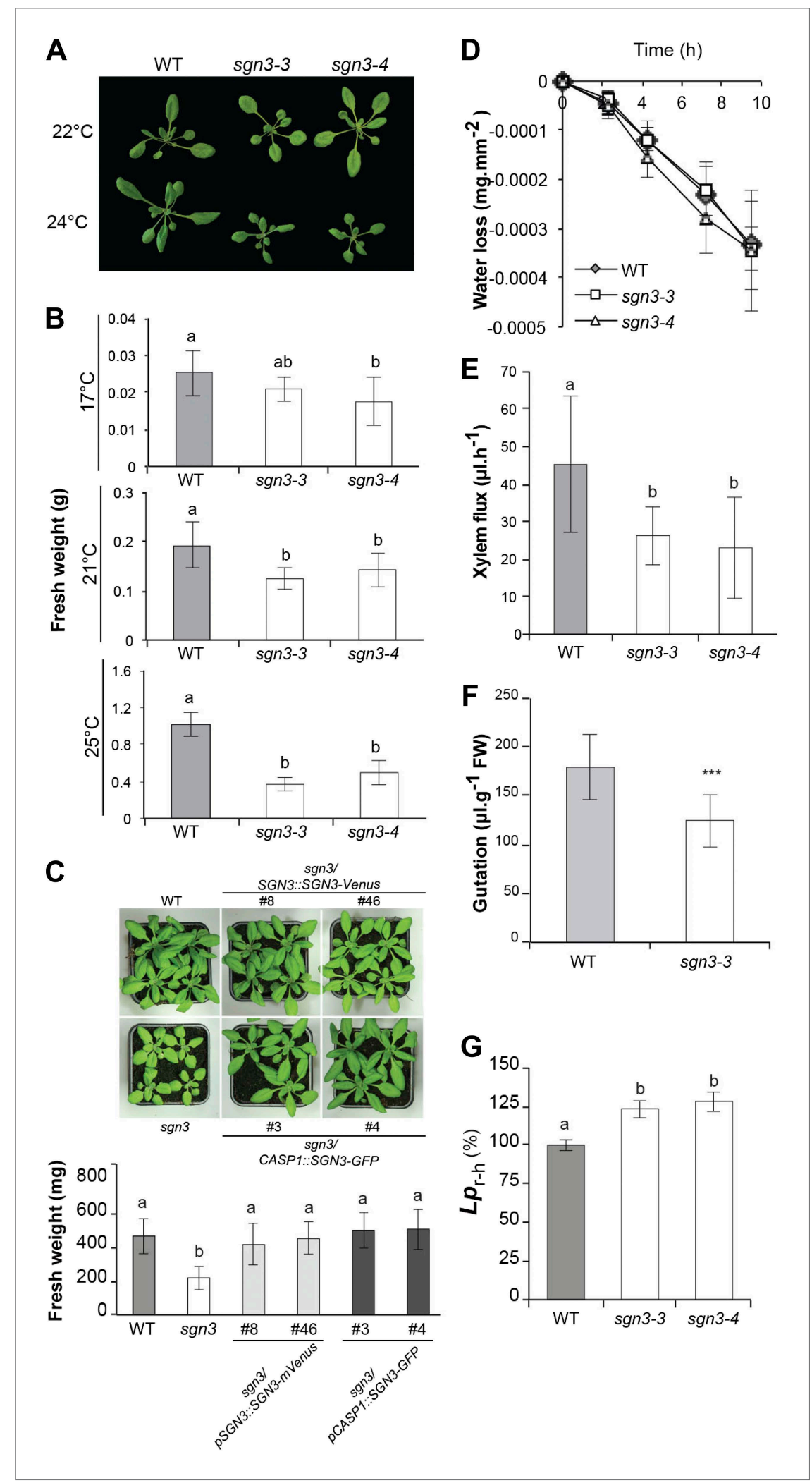

Figure 4. sgn3 is sensitive to environmental conditions and displays an altered water transport and root pressure. (A) Phenotype of 3-week-old WT, sgn3-3, and sgn3-4 plants grown at 22 or $24^{\circ} \mathrm{C}$ in long days. Representative pictures are presented. (B) Analysis of shoots fresh weight of WT, sgn3-3, and sgn3-4 plants ( $n=15)$ grown 3 weeks Figure 4. Continued on next page 
Figure 4. Continued

at different temperatures $\left(17,21\right.$, or $\left.25^{\circ} \mathrm{C}\right)$. (C) Phenotype of 4-week-old WT, sgn3-3, sgn3-3/pSGN3::SGN3-mVenus (lines 8 and 46), and sgn3-3/pCASP1::SGN3-GFP (lines 3 and 4) grown at $24^{\circ} \mathrm{C}$ in long days. Representative pictures are presented. Fresh weight average from $n>8$ plants. (D) Transpiration of WT, sgn3-3, and sgn3-4 plants determined as water loss from 3-week-old plants. Error bars = s.d. $(n=10)$. (E) Root pressure analysis determined as the volume of xylem sap released in 30 min from decapitated WT, sgn3-3, and sgn3-4 plants grown in short day condition $(n=7)$. ( $F)$ Guttation was collected from WT and sgn3-3 plants grown for 6 weeks in short day conditions ( $n=15)$. (G) Mean hydrostatic hydraulic conductivity of roots $\left(L_{r-h}\right)$ from WT, sgn3-3, and sgn3-4 plants. $L p_{r-h}$ was measured during the daytime. Values correspond to means $\pm \mathrm{SD}(\mathrm{n}>14)$. B, C, E, F, G. Error bars $=$ s.d. For multiple comparison, different letters indicate significant differences between genotypes, determined by analysis of variance (ANOVA) and Tukey test as post hoc analyses; (B, $p<0.01)(\mathbf{E}, \mathbf{G}, \mathrm{p}<0.05)$. For single comparison in $\mathbf{F}$, stars $\left(_{* * *}\right)$ indicate significant difference determined by Student test $(p<0.001)$.

DOI: 10.7554/eLife.03115.012

The following figure supplement is available for figure 4:

Figure supplement 1. Impact of environmental conditions on growth of sgn3 mutant. DOI: 10.7554/eLife.03115.013

plants that did not reproduce when grown in different, standard growth chambers that all well supported wild type growth. We wanted to ascertain that the observed growth phenotypes of sgn3 are indeed caused by the Casparian strip defect in the roots and not by some hypothetical, non-redundant function of SGN3 in shoots. We therefore expressed SGN3 under the control of the CASP1 promoter that shows specific expression in differentiating root endodermis, with some expression in the endodermis of the lower hypocotyl and none detectable anywhere else in the plant. These CASP1::SGN3-GFP sgn3 plants could fully rescue the growth phenotype of sgn3 plants (Figure 4C), demonstrating that it is the root endodermal defect of sgn3 that is causative for the overall growth defect of the mutant.

\section{sgn 3 mutants display normal transpiration but altered water transport and root pressure}

The numerous, repeated Casparian strip discontinuities in sgn3 must generate an apoplastic bypass for both water and solutes in the mutant root. Especially under conditions of high transpiration, this would be expected to lead to a strong, uncontrolled and possibly detrimental influx of elements into the transpiration stream. We observed that transpiration rate per surface area in sgn3 mutants are very similar to wild type (Figure 4D) revealing that there is no compensatory downregulation of transpiration. Another predicted consequence of interrupted Casparian strips is an inability to build up root pressure, a process necessary for vascular transport of nutrients and water in the absence of transpiration and for transport into non-transpiring organs (Wegner, 2014). Root pressure build-up in the dead xylem vessels is thought to involve an osmotic gradient, generated by living neighbors, that draws water into the vessels. Pressure build-up, however, is thought to crucially depend on the presence of the Casparian strips as an apoplastic diffusion barrier, without which xylem pressure should quickly dissipate into the cortex. We indeed found that root pressure, as estimated by xylem sap exudation from hypocotyls after decapitation, was lower in the sgn3 mutant (Figure 4E). sgn3 also showed a decreased rate of leaf guttation, a process more indirectly dependent on root pressure, but that can be observed less invasively (Figure 4F). As a more direct measure of apoplastic water flow, we inserted excised roots in a pressure chamber and found a significant increase in root hydrostatic hydraulic conductivity $\left(L p_{r-h}\right)$ in sgn3 mutants compared to wild type (Figure $4 G$ ). The defects of Casparian strip formation may allow an apoplastic bypass for water in pressurized sgn3 roots thereby explaining its increased $L p_{r-h}$. Our findings are the first genetic evidence that Casparian strips can be relevant for root pressure buildup and impose a hydraulic resistance; the magnitude of the observed effects nevertheless indicates that an intact Casparian strip is not an absolute requirement and that unmodified walls and/or suberized cells can generate a sufficiently high resistance to radial waterflow so that the positive root pressure can be maintained. sgn3 might be very useful to dissect the mechanisms of root pressure formation and its role in nutrient transport in the future.

\section{Transcriptional profiles suggest a latent potassium deficiency in sgn3 mutants}

In order to identify some potential stress and/or compensatory responses in the sgn 3 mutant, we generated a general transcriptional profile of sgn3 mutant rosettes, grown under conditions in 
which wild type and sgn 3 were indistinguishable. Only little differences between sgn3 and wild type were observed. Yet, all of the seven more highly expressed genes in sgn3 were found to be also induced by potassium starvation, suggesting a latent, weak potassium deficiency stress under these conditions (Figure 5A, Figure 5-source data 1). Based on this indication, we tested well-established transcriptional read-outs of potassium deficiency in roots, the potassium influx transport proteins AKT1 and HAK5, and found that both were upregulated in sgn3 (Figure 5B,C).

\section{An impaired endodermal barrier causes surprisingly specific defects in elemental homeostasis}

In order to directly measure how sgn3 root barrier defects affect elemental homeostasis in leaves, we undertook a number of elemental profiling experiments using inductively coupled plasma-mass spectrometry (ICP-MS) on rosette leaves in different laboratories under different growth conditions. Surprisingly, concentrations of most elements remained essentially unaltered in sgn3. Despite the dramatic apoplastic bypass in sgn3 roots, the mutants managed to maintain wild type levels of sodium $(\mathrm{Na})$, calcium (Ca), or boron (B), for example. Among the measured transition elements (Mo, Co, Mn, $\mathrm{Zn}, \mathrm{Fe}, \mathrm{Cu}, \mathrm{Zn}$ ) only zinc showed a significant decrease in some measurements (Figure 5D, Figure 5source data 2). However, matching the transcriptional profiling results, we invariably found lower levels of potassium $(K)$ in the mutant (ranging from 1.4-3.0 fold reduction). Inversely, levels of magnesium (Mg) and cesium (Cs) accumulated to higher levels than in wild type, ranging from 1.5-2.1 and 1.3- to 1.4-fold increase, respectively (Figure 5D, Figure 5-source data 2). These findings nicely corroborated the results of our microarrays, but left us wondering about the absence of potassium deficiency symptoms in sgn3, since the mutant phenotypes under most conditions are simply those of a reduction and delay in growth.

\section{The sgn 3 mutant is strongly hypersensitive to low potassium conditions}

We then tested growth of sgn3 plants on a nutrient-poor, gravel-like substrate, which we watered with a nutrient solution with or without potassium. Wild type plants coped well with conditions of low potassium nutrition, with most plants displaying no or only very weak chlorosis on older rosette leaves, indicative of potassium deficiency (Figure 5E,F). Consistently, wild type also maintained higher potassium concentration than sgn3 under these conditions (Figure 5-figure supplement 1). The sgn 3 mutant in contrast, developed severe chlorosis of its rosette leaf margins, characteristic of strong potassium deficiency (Marschner and Marschner, 2012). sgn3 therefore has an impaired capacity to accumulate sufficient potassium under conditions of low supply of this element.

\section{Discussion}

\section{The SGN3 receptor-like kinase is important for microdomain organization and enhanced suberization in the endodermis}

The deposition of cell wall material with subcellular precision is crucial for the correct function of many plant cell types (Roppolo and Geldner, 2012). How this localized deposition is achieved is only rarely understood in any mechanistic detail, with the notable exception of wall deposition in metaxylem cells (Oda and Fukuda, 2012). Localized wall deposition should implicate the formation of microdomains in the plasma membrane that localize cell wall biosynthetic activity. Walls, in turn, signal back to the cell, and this process informs about mechanical stresses, wall polymer breakdown or for regulated expansion growth (Cheung and $W u, 2011$; Lindner et al., 2012). The precisely localized, ring-like Casparian strip can be used as a model for studying localized wall deposition (Lee et al., 2013). The SGN3 LRR-RLK appears to be involved in both the formation of the CSD and the signaling of defective Casparian strips. Lack of SGN3 causes an inability of CASP1-GFP patches to fuse into a contiguous band, without affecting the positioning of the patches along a median ring, nor the stability or functionality of the already established patches. SGN3 localization to the transversal and anticlinal, but not the periclinal membrane domains further increases the complexity of subdomains present at the endodermal plasma membrane. It embeds the forming CASP domain into a larger subdomain within which the narrower CSD is formed. Direct interaction of CASPs and SGN3 could take place early during CASP accumulation at the membrane and occur at the rims of forming CASP patches, possibly promoting patch growth and fusion. This can now be tested in future experiments. A second, currently unrelated role for SGN3 is the induction of increased lignin 
A

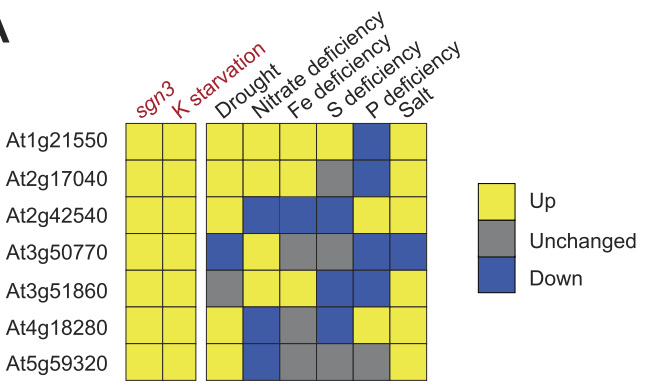

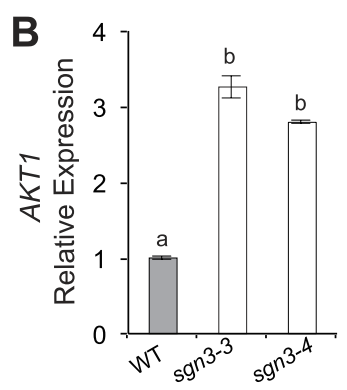

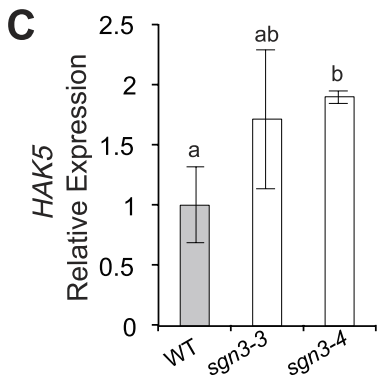

D

\begin{tabular}{|c|c|c|c|c|c|c|c|c|c|c|c|c|c|c|c|c|}
\hline Growth condition & Experiment & Mutant & B & $\mathrm{Ca}$ & Co & $\mathrm{Cu}$ & Cs & $\mathrm{Fe}$ & $\mathrm{K}$ & $\mathrm{Mg}$ & $\mathrm{Mn}$ & Mo & $\mathrm{Na}$ & $\mathrm{P}$ & $\mathrm{Sr}$ & $\mathrm{Zn}$ \\
\hline \multirow{4}{*}{ hydropony, short days } & & $\operatorname{sgn} 3-3$ & & & & & & & & & & & & & & \\
\hline & Hokkaido 06/13 & $\operatorname{sgn} 3-4$ & & & & & & & & & & & & & & \\
\hline & Hokkaido $08 / 13$ & $\operatorname{sgn} 3-3$ & & & & & & & & & & & & & & \\
\hline & & $\operatorname{sgn} 3-4$ & & & & & & & & & & & & & & \\
\hline \multirow{2}{*}{ hydropony, long days } & \multirow{2}{*}{ Lausanne 05/12 } & $\operatorname{sgn} 3-3$ & n.d. & n.d. & n.d. & & n.d. & & & n.d. & & n.d. & n.d. & n.d. & n.d. & \\
\hline & & $\operatorname{sgn} 3-4$ & n.d. & n.d. & n.d. & & n.d. & & & n.d. & & n.d. & n.d. & n.d. & n.d. & \\
\hline \multirow{2}{*}{ soil, long days } & \multirow{2}{*}{ Lausanne 03/11 } & $\operatorname{sgn} 3-3$ & n.d. & & n.d. & n.d. & n.d. & n.d. & & & n.d. & n.d. & & n.d. & n.d. & n.d. \\
\hline & & $\operatorname{sgn} 3-4$ & n.d. & & n.d. & n.d. & n.d. & n.d. & & & n.d. & n.d. & & n.d. & n.d. & n.d. \\
\hline soil, short days & Aberdeen 03/12 & $\operatorname{sgn} 3-19$ & & & & & n.d. & & & & & & & & & \\
\hline
\end{tabular}

Up Unchanged Down

E

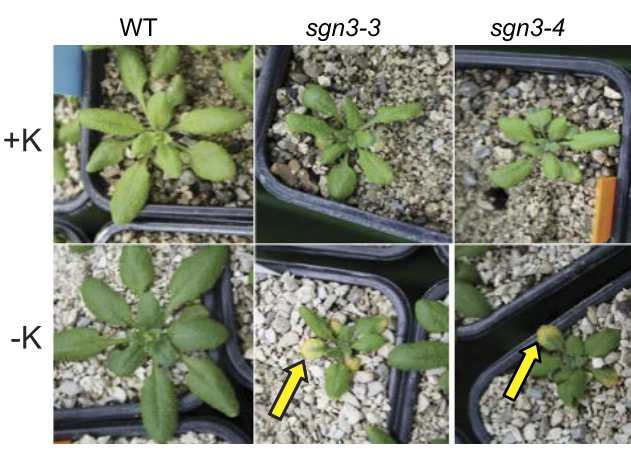

$\mathbf{F}$

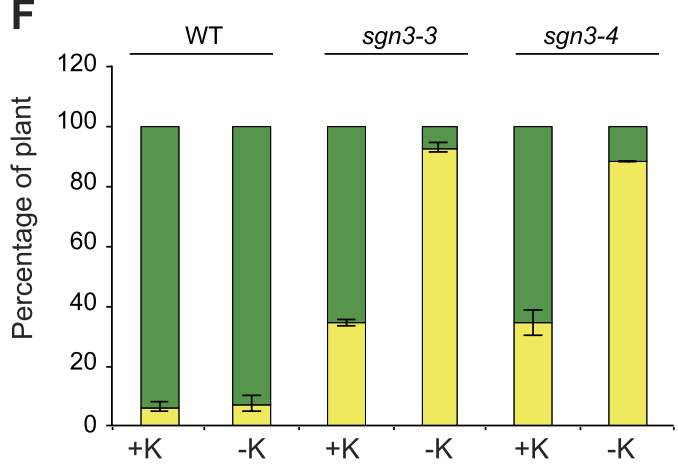

Figure 5. Potassium homeostasis is affected in sgn3. (A) Expression of genes upregulated in 4 week-old sgn3 leaves. The seven genes presented here are the ones whose expression level was significantly increased in sgn3 $(p<0.15)$. Those genes were investigated in Genevestigator for responses in leaves to nutritional stresses such as potassium starvation, drought, nitrate deficiency, Fe deficiency, S deficiency, P deficiency, and salt stress. Color-code indicates the effect of growth condition to their expression level (yellow up, gray unchanged, blue down). For numerical values see Figure 5-source data 1. (B and C) Quantitative RT-PCR analysis of AKT1 (B) and HAK5 (C) transcript levels in WT, sgn3-3, and sgn3-4 roots ( $n=3$ ). Error bars = s.d, different letters indicate significant differences between genotypes, determined by analysis of variance (ANOVA) and Tukey test as post hoc analyses $(p<0.05)$. (D) Overview of ionomic analysis performed in sgn3 leaves (sgn3-3, sgn3-4, and sgn3-19 alleles) in three independent laboratories (Hokkaido, Lausanne, Aberdeen), using 2 growth systems (hydroponics or soil) and 2 day-length conditions (short or long days). Elements were determined by ICP-MS (Hokkaido, Lausanne 05/12 and Aberdeen) or ion chromatography (Lausanne 03/11). Color-code indicates significant changes of accumulation in sgn3 mutants compared to WT ( $p<0.05$; yellow up, gray unchanged, blue down). For the numerical values, see Figure 5-source data 2. (E and F) Phenotype of 3-week-old WT, sgn3-3, and sgn3-4 plants grown in potassium (K) deficiency. Plants were watered from germination with a nutritive solution with or without $1.5 \mathrm{mM} \mathrm{KNO}_{3}(+\mathrm{K}$ or $-\mathrm{K})$. Representative picture are presented. Arrow indicates a chlorotic leave. (F) Occurrence of K deficiency phenotype determined as the percentage of plants displaying at least one yellow leaf (yellow) vs percentage of plants displaying only green leaves (green). Error bars = s.d.; data correspond to the mean of two independent experiments with a total of $\mathrm{n} \geq 50$ plants.

DOI: 10.7554/eLife.03115.014

The following source data and figure supplements are available for figure 5 :

Source data 1. Transcriptional differences between wild type and sgn3 shoots.

DOI: 10.7554/eLife.03115.015

Figure 5. Continued on next page 
Figure 5. Continued

Source data 2. Overview of ionomic experiments.

DOI: 10.7554/eLife.03115.016

Figure supplement 1. Ionomic comparision of WT and sgn3 grown under low potassium.

DOl: 10.7554 /eLife.03115.017

and suberin formation in other Casparian strip mutants, such as esb1 or casp1 casp3. We speculate that SGN3 acts as a kinase that reports CSD integrity, signaling an upregulation of lignin, and suberin production. A surveillance of Casparian strip integrity could be of great importance for unimpeded root function and would parallel the surveillance of tight junctions that has been reported for animal epithelia (Balda and Matter, 2009).

\section{SGN3 has redundant activities outside the endodermis}

We could demonstrate that exclusive expression of SGN3 in the differentiating root endodermis is sufficient to rescue the overall growth defect of sgn3. This indicates that the endodermal function that we describe here is the only specific, non-redundant activity of this receptor in the plant. The earlier described function of SGN3/GSO1 in the formation of an embryonic cuticle by contrast is fully redundant with that of its homolog GSO2. The dramatic cotyledonary phenotype of the double mutant is bound to have secondary consequences on seedling growth, making it very difficult to dissect primary from secondary effects. A recent investigation of root meristem defects of the double mutants, for example, could entirely be explained as secondary consequences of the primary defect in embryonic cuticle formation (Racolta et al., 2014). It is nevertheless important to speculate how the two functions of endodermal Casparian strip formation and protodermal cuticle formation could be explained by a common molecular activity. By genetic analysis, the GSO1 GSO2 pair has been assigned to a signaling pathway that depends on an endosperm-expressed subtilase (ABNORMAL LEAF-SHAPE1, ALE1) (Xing et al., 2013). In a currently speculative scenario, subtilases might be involved in processing of peptide hormones that could act as ligands for SGN3/GSO1, promoting formation of a functional cuticle. Yet, the endodermal, lignified Casparian strip and the protodermal, cutin-based embryonic cuticle are two chemically different cell wall modifications that also show distinct modes and sites of deposition within the primary cell wall. These differences make it difficult to perceive a common denominator for SGN3 action in these two cell types. Moreover, our data suggest that a primary function of SGN3/GSO1 is the formation of a continuous band of CASP membrane proteins. No CASP homologs have been implicated in cuticle formation up-to-now, nor are there any indications for the presence of a highly scaffolded membrane protein domain in the epidermis/protodermis that would resemble the CSD. Another SGN3/GSO1 function that we could establish is the promotion of suberin formation in response to a defective Casparian strip. Enhanced suberin formation in esb1 appears to be entirely dependent on SGN3 activity, although the normal developmental progression of suberin formation is not affected in the mutant. Suberin and cutin are chemically closely related polymers whose monomers are formed by sets of homologous enzymes. A common role of SGN3 might be to mediate a cross-talk between cell wall components, possibly regulating suberin production in endodermis and cutin production in epidermis/protodermis. Identification of the SGN3 ligands and downstream kinase substrates will be crucial for an understanding of the common mechanism that might underlie these apparently divergent functions.

\section{sgn 3 is the strongest and most specific Casparian strip mutant known to date}

Regardless of the fact that SGN3 has other roles in the plant, the sgn3 single mutant defect reported here remains exquisitely specific to the endodermis. This is supported by the fact that it is only in the endodermis where SGN3 expression is non-overlapping with its close homolog, GSO2 (Tsuwamoto et al., 2008). Moreover, exclusive expression of SGN3 in the endodermis is able to rescue the overall growth defects observed in aerial parts (this study). In addition to its specificity, the sgn3 phenotype turns out to be strong and robust, that is, it displays penetration of tracer along the whole length of a seedling root and the phenotype is stable in the different growth conditions that we tested. Finally, sgn3 does not show any of the enhanced suberin formation that has been observed in other mutants and which confounds interpretation of phenotypes. We therefore propose that the sgn3 mutant is uniquely suited to obtain insights into the specific role of Casparian strips in plants. This mutant might be very 
useful for investigating not only root nutrient and water uptake, but also for addressing the role of the endodermal barrier in pathogen resistance, hormonal transport, stress, or general growth responses.

\section{sgn3 nutrient homeostatic defects are surprisingly specific}

Our first analysis of the sgn3 mutant has revealed an unexpected robustness of Arabidopsis growth towards a complete lack of its main root diffusion barrier. While still able to grow and complete its life cycle, the mutant nevertheless displays greatly exacerbated growth reductions to non-stressful changes in environmental conditions. This indicates the presence of homeostatic backup systems in the plant that are incompletely compensating for the absence of Casparian strips. Homeostasis of many elements is kept in the sgn3 mutant, and it is intriguing that it is specifically potassium that shows a reproducibly strong decrease in concentration. Potassium $(K)$ is the only macronutrient $(N, P, K, S)$ that is not integrated into polymers and that remains highly mobile within the plants. It is being used as a counter ion in many transport process and is recycled back to the root along with the phloem sap. At the same time, concentrations of potassium required for plant growth are high and potassium often needs to be concentrated 10-100 fold over the concentration found in the soil (Marschner and Marschner, 2012; Wang and Wu, 2013). It therefore makes a lot of sense that potassium is an element that cannot be kept at sufficiently high levels in Casparian strip defective plants. We propose that potassium located in the stelar apoplast is continuously lost to the outer cell layers and the soil in the absence of functional Casparian strips. Upregulation of potassium influx carriers in cortex/epidermis, as we have observed in sgn 3 roots, would certainly be able to recuperate large parts of the potassium being lost. However, a chronic condition of very low external potassium would lead to a breakdown in this transporter-mediated 'potassium recycling' and eventually cause the deficiency phenotypes that we observe. From this scenario, it follows that there should be a higher rate of potassium flux out-of and into the root stele in a sgn 3 mutant and that potassium homeostasis in the mutant is extremely dependent on increased activity of potassium transporters. We are currently working on substantiating this 'potassium recycling' model by specific interference with and visualization of potassium transporters in the mutant. Further analysis of sgn3 will be instructive for a better understanding of root potassium transport in general and this analysis should also be extended to other macronutrients in the future.

What could be the reasons for the lack of effects on many other elements? It can be expected that similar compensatory responses as seen for potassium exist for other elements. If these elements need to be less concentrated over their external concentration-or if they are less mobile within the plant-the compensatory responses might be sufficient for maintaining homeostasis in the mutant. Interestingly, we found an increase in the levels of the divalent cation magnesium, while concentrations of the divalent calcium, for example, were not affected in sgn3 mutants. This could be explained by a lower concentration gradient between the xylem sap and the external environment and a lower mobility of Calcium within the cell wall space (Clarkson, 1984; White and Broadley, 2003). Nevertheless, calcium concentration within the symplast is kept extremely low for cellular signaling purposes. Therefore, calcium delivery to aerial tissues is thought to occur either entirely through the apoplast-using the few apoplastic 'bypasses' of the endodermis at the root tip or at lateral root emergence sites (White, 2001)—or to employ a very short symplastic route passing exclusively through endodermal cells (Clarkson, 1984). In both scenarios, it is surprising that the opening of a massive apoplastic bypass in the sgn3 mutant has no effect on calcium homeostasis, while it did affect levels of magnesium. Possibly, magnesium is more mobile in the apoplast than Calcium and undergoes increased apoplastic transport into the transpiration stream in sgn3 (Thibault and Rinaudo, 1985). Identifying conditions in which calcium homeostasis also breaks down in sgn3 would greatly advance our understanding of the calcium uptake mechanisms in plants.

The sgn3 mutant represents a powerful new tool to better understand root uptake and transport mechanism of most other plant nutrients as well. Our initial analysis of the sgn 3 mutant challenges the current notions of the role of the Casparian strip as a required barrier for most or all elements. The ability to selectively disrupt this strict endodermal barrier will allow for direct investigations of other mechanisms that maintain and buffer ion homeostasis. Unmodified cell walls, by their properties as ion exchange matrices, in conjunction with vacuolar storage of elements, can play pivotal roles in maintaining homeostasis of many elements. Cytoplasmic magnesium levels, for example, might well remain unaltered in sgn3, the increased magnesium levels being entirely absorbed by the vacuole. Having taken away the 'first lock' of the Casparian strip now puts the focus on the existence of 'second locks' that can be studied using the sgn 3 mutant as a sensitized background. 


\section{Materials and methods}

\section{Plant material}

Arabidopsis thaliana ecotype Columbia was used for all experiments. For details of knockout mutants, see Table 1.

\section{Vector construction and transgenic lines}

For generation of expression constructs, Gateway Cloning Technology (Invitrogen, Carlsbad, CA) or standard molecular biology procedures were used. pESB1::ESB1-mCherry plasmid (Hosmani et al., 2013) was transformed in sgn3-3 background. pSGN3 (5583 bp before ATG)::SGN3 gDNA-mVenus was cloned by integrating a 9.4-kb genomic fragment including the intron and the 5'UTR into a Bastaresistance $p$ GREENII vector containing mVenus. pCASP1::NLS-GFP-GUS and pCASP4::NLS-GFP-GUS reporters were cloned into a Basta-resistance pGREENII vector and contain 1048 bp and 695 bp promoter fragments, respectively. pCASP1::SGN3 cDNA-GFP was cloned with Gateway using a 1207 promoter fragment followed by SGN3 cDNA without the unique intron and GFP. pCASP1 (1207 bp)::PDR6 gDNA-Venus-4G (4 glycine extension) was cloned using Gateway in a pB7m34 GW,3 expression vector (http://gateway.psb.ugent.be). pCASP1 (1207 bp)::mCherry-SYP122 was constructed with Gateway by cloning the AtSYP122 (At3g52400) cDNA into pH7m42 GW,3. pCASP1::CASP2-GFP-4G, pCASP1::CASP3-GFP-4G, pCASP1::CASP4-mCherry and pCASP1::CASP5GFP-4G were cloned with Gateway using the pB7m34 GW,3 expression clone. Transgenic plants were generated by introduction of the plant expression constructs into an Agrobacterium tumefaciens strain GV3101 and transformation was done by floral dipping (Clough and Bent, 1998).

\section{Growth conditions}

For in vitro assays, plants were germinated on 0.5 MS (Murashige and Skoog) agar plates after 2 days in dark at $4^{\circ} \mathrm{C}$. Seedlings were grown vertically in growth chambers at $22^{\circ} \mathrm{C}$, under long days $(16-\mathrm{hr}$ light/8-hr dark), $100 \mu \mathrm{E}$ light, and were used at 5 days after shift to room temperature. For microarray, transpiration, hydraulic conductivity, and ionomic analysis in Lausanne (May 2012) assays, plant were germinated on hydroponic conditions after 2 days in dark at $4^{\circ} \mathrm{C}$. Nutrient solution was changed weekly and contained $0.5 \mathrm{mM} \mathrm{KNO}_{3}, 0.25 \mathrm{mM} \mathrm{Ca}\left(\mathrm{NO}_{3}\right)_{2}, 1 \mathrm{mM} \mathrm{KH}_{2} \mathrm{PO}_{4}, 1 \mathrm{mM} \mathrm{MgSO}_{4}, \mathrm{FeNH}_{4}-\mathrm{EDTA}$ $0.1 \mathrm{mM}, \mathrm{KCl} 50 \mu \mathrm{M}, \mathrm{H}_{3} \mathrm{BO}_{3} 30 \mu \mathrm{M}, \mathrm{MnSO}_{4} 5 \mu \mathrm{M}, \mathrm{ZnSO}_{4} 1 \mu \mathrm{M}, \mathrm{CuSO}_{4} 1 \mu \mathrm{M},\left(\mathrm{NH}_{4}\right)_{6} \mathrm{Mo}_{7} \mathrm{O}_{24} 0.1 \mu \mathrm{M}$. Light/dark cycle was $16 \mathrm{hr} / 8 \mathrm{hr}, 23^{\circ} \mathrm{C} / 18^{\circ} \mathrm{C}, 100 \mu \mathrm{E}$ light.

For xylem sap and guttation assays, plants were germinated after 2 days in dark at $4^{\circ} \mathrm{C}$ on soil in short day condition and irrigated with water. Light/dark cycle was $10 \mathrm{hr} / 14 \mathrm{hr}, 22^{\circ} \mathrm{C} / 18^{\circ} \mathrm{C}, 100 \mu \mathrm{E}$ light.

For phenotypic assays at different light intensities $(30,90,150$, or $300 \mu \mathrm{E})$, temperatures $(17,21$, or $25^{\circ} \mathrm{C}$ ), or day lengths $(8,16$ or $24 \mathrm{hr})$, plants were germinated after 2 days in dark at $4^{\circ} \mathrm{C}$ on soil and irrigated with water. The standard condition was: light/dark cycle $16 \mathrm{hr} / 8 \mathrm{hr}, 21^{\circ} \mathrm{C} / 19^{\circ} \mathrm{C}$ with light intensity of $150 \mu \mathrm{E}$. Temperature difference between day and night was always kept at $2^{\circ} \mathrm{C}$.

Table 1. Details of knockout mutants

\begin{tabular}{|c|c|c|c|c|c|}
\hline Gene number & Accession & Mutant number & Mutant name & Genotyping primer sequence & References \\
\hline AT4G20140 & Col-0 & SALK_043282 & $\operatorname{sgn} 3-3$ & $\begin{array}{l}\text { LP: ATTCTACGAGCCTTCCCATTC } \\
\text { RP: CGCAGTGAACACAGTGAGATC }\end{array}$ & Present work \\
\hline AT4G20140 & Col-0 & SALK_064029 & sgn3-4 or gso1-1 & $\begin{array}{l}\text { LP: CTCGGCTCCCTCGTTAATATC } \\
\text { RP: GTTACCTAAACTGGCGGGAAG }\end{array}$ & $\begin{array}{l}\text { Tsuwamoto et al. (2008) } \\
\text { The Plant Journal }\end{array}$ \\
\hline AT4G20140 & Col-0 & SALK_103965 & sgn3-19 & $\begin{array}{l}\text { LP: TCCATTATGTGGTTCGAGCTC } \\
\text { RP: CTTGTAAACCTTCCCAGAGCC }\end{array}$ & Present work \\
\hline AT2G28670 & Col-0 & n.a. & esb1-1 & n.a. & $\begin{array}{l}\text { Baxter I et al. (2009) PLOS Genet, } \\
\text { Lahner B et al. (2003) Nat Biotechnol }\end{array}$ \\
\hline AT2G36100 & Col-0 & SAIL_265_H05 & casp1-1 & $\begin{array}{l}\text { LP: GCGTTTCAGTACGTCCCTTC } \\
\text { RP: CACGTGAGGGAAGTGAGTCTC }\end{array}$ & Roppolo et al. (2011) Nature \\
\hline AT2G27370 & Col-0 & SALK_011092 & casp3-1 & $\begin{array}{l}\text { LP: GACTCTTCCTTTCTTCACTC } \\
\text { RP: GACCAACACAACCGTACGAAC }\end{array}$ & Roppolo et al. (2011) Nature \\
\hline
\end{tabular}


For ionomic assays plants were grown in different laboratories as indicated. For the Hokkaido experiments plants were grown hydroponically as described in Takano et al. (2001), with slight modifications. Environmental parameters in a growth chamber are as follows: 10-hr/14-hr light/dark cycle, $22^{\circ} \mathrm{C}$ under fluorescent lamps, $70 \%$ humidity. The seeds were sown on rockwool and grown supplied with hydroponic media (Fujiwara et al., 1992) supplemented with $50 \mu \mathrm{M}$ Fe-EDTA. After 15 days, the plants were additionally supplied with $3 \mu \mathrm{M} \mathrm{CsCl}$ and $10 \mu \mathrm{M} \mathrm{SrCl}_{2}$. The media were changed twice a week. For the Aberdeen experiment plants were grown in short day conditions, in Bulrush multipurpose compost (http://www.bulrush.co.uk/retail-range/all-purpose-composts.html) spiked with various elements as detailed in Lahner et al. (2003), and bottom watered using $1 / 4$ Hoagland solution containing $\mathrm{Fe}$ as $10 \mu \mathrm{M} \mathrm{Fe}$-HBED as described in Baxter et al. (2008). 1-2 adult rosette leaves were harvested 36 days after planting. For the Lausanne experiment in soil (March 2011), plants were grown in long days 16 -hr/8-hr light/dark, $23^{\circ} \mathrm{C} / 19^{\circ} \mathrm{C}$, and leaves were harvested after 5 weeks.

For potassium deficiency assay plants were germinated after 2 days in dark at $4^{\circ} \mathrm{C}$ on a poor gravellike substrate (OIL DRI US-Special) watered with a 1/2 Hoagland based solution containing : $1.5 \mathrm{mM}$ $\mathrm{NH}_{4} \mathrm{NO}_{3}$ or $1.5 \mathrm{mM} \mathrm{KNO}{ }_{3}(-\mathrm{K}$ or $+\mathrm{K})$.

\section{Confocal microscopy}

Confocal laser scanning microscopy experiments were performed either on a Leica SP2, a Zeiss LSM 700, a Zeiss LSM 710, or a Zeiss LSM 710 NLO 2-Photon microscope. Excitation and detection windows were set as follows: Leica SP2: GFP $488 \mathrm{~nm}, 500-600 \mathrm{~nm}$; propidium iodide $488 \mathrm{~nm}, 600-700$. Zeiss LSM 700: GFP/mVenus $488 \mathrm{~nm}$, 490-555 nm; mCherry/propidium iodide $555 \mathrm{~nm}$, SP 640. LSM 710: GFP/mVenus $488 \mathrm{~nm}, 495-554 \mathrm{~nm}$; mCherry/propidium iodide $561 \mathrm{~nm}, 573-681 \mathrm{~nm}$. Zeiss LSM 710 NLO 2-Photon: GFP/mVenus, $960 \mathrm{~nm}, 500-550 \mathrm{~nm}$ (NDD). For visualization of the apoplastic barrier, seedlings were incubated in the dark for $10 \mathrm{~min}$ in a fresh solution of $15 \mathrm{mM} \mathrm{(10} \mathrm{mg/ml)} \mathrm{Propidium}$ lodide (PI) and rinsed two times in water (Alassimone et al., 2010, Naseer et alo, 2012). For quantification, 'onset of elongation' was defined as the point where an endodermal cell in a median, longitudinal section reached a length more than twice its width. From this point, cells in the file were counted until the PI signal was blocked in the endodermal cells. For plants grown in hydroponic and in soil, roots of 13 and 14 days were used, respectively.

Casparian strip autofluorescence after clearing was visualized as described (Alassimone et alo, 2010, Naseer et al., 2012).

Fluorescence recovery after photobleaching (FRAP) was performed with 5-day-old seedlings and imaged with a Leica SP2 confocal microscope as described (Roppolo et al., 2011).

Plasmolysis was induced by incubating 5-day-old seedlings for $1 \mathrm{hr}$ in $0.8 \mathrm{M}$ mannitol and then mounted in the same solution. Plasmolyzed cells were imaged at 15 cells after the onset of CASP1 expression.

For time-lapse imaging of CASP1::CASP1-GFP in Col-0 and sgn3-3, 5-day-old seedlings were into a Lab-Tek II chambered coverglass (Nunc) and covered with a small block of agar to prevent drying. Subsequently, slides were mounted and imaged on an upright Zeiss LSM710 NLO confocal microscope. Excitation was provided by a Ti-Sapphire Chameleon II Ultra (Coherent) with $960 \mathrm{~nm}$ and fluorescence was detected using non-descanned detection (NDD) between 500 and $550 \mathrm{~nm}$. Image stacks were taken every $15 \mathrm{~min}$ for a total time of $16 \mathrm{hr}$. Confocal images were analyzed and contrast and brightness were adjusted with the FIJl package (http://fiji.sc/Fiji) and Adobe Photoshop CS5.

For the percentage quantification of Casparian strips, autofluorescence after clearing was performed on ten seedlings for each genotype investigated. For each individual seedling, Casparian strips were imaged by confocal microscopy by doing one z-stack and measurements were done using ImageJ. The percentage of Casparian strip was the portion where autofluorescence was visible along a line showing Casparian strip signal. A minimum of $3.5 \mathrm{~mm}$ of Casparian strip was quantified in each genotype.

\section{Suberin lamellae analysis}

Suberin lamella was observed in 5-day-old roots after Fluorol Yellow staining as described in Naseer et al. (2012). Seedlings were incubated in Fluorol Yellow $088(0.01 \% \mathrm{wt} / \mathrm{vol}$, lactic acid $)$ at $70^{\circ} \mathrm{C}$ for $30 \mathrm{~min}$, rinsed with water, and counterstained with aniline blue ( $0.5 \% \mathrm{wt} / \mathrm{vol}$, water) at RT for $30 \mathrm{~min}$ in darkness, washed, mounted on slides with glycerol and observed with epifluorescence microscope. Suberin pattern were observed and counted from the hypocotyl junction to the onset of endodermal cell elongation. Three distinct patterns were considered: continuous suberin lamellae, discontinuous 
suberin lamellae (corresponding to the area where suberin lamellae establish), and non- suberized (corresponding to the young part of the root). Experiments were repeated at least four times.

\section{Water transport assay}

\section{Transpiration}

For transpiration assays, 3-week-old plants grown in hydroponic conditions were transferred to airtight containers isolating the root and nutritive solution from the air while plant shoots remain out of the container (potometer). Transfer to potometer was performed $24 \mathrm{hr}$ prior to transpiration measurement. Transpiration was determined as the loss of weight measured every $2 \mathrm{hr}$ during $14 \mathrm{hr}$ and was expressed relatively to the plant's leaf area. Leaf area was measured for each individual plant, with ImageJ from pictures of detached leaves at the end of the transpiration assay.

\section{Root pressure}

Plants were grown 6 weeks in short day condition and decapitated with scalpel at the hypocotyl junction. The decapitated hypocotyl was immediately introduced into a $100 \mu \mathrm{l} \mathrm{microcapillary.} \mathrm{Root}$ pressure was determined as the volume of xylem sap collected within 30 min from decapitated plants.

\section{Guttation}

Plants were grown 6 weeks in short day condition. To induce guttation, plants were transferred to $15^{\circ} \mathrm{C}$ in the dark for $12 \mathrm{hr}$, covered with a lid to maintain high humidity. The liquid was harvested from 15 individual plants for each genotype, using a micropipette.

Hydrostatic hydraulic conductivity

Measurements of root hydrostatic hydraulic conductivity $\left(L \mathrm{p}_{\mathrm{r}-\mathrm{h}}\right)$ were performed as described previously (Javot et al., 2003; Postaire et al., 2010).

\section{lonomic analysis}

For ionomic assays plants were grown in different laboratories as indicated. For the Hokkaido and Lausanne experiments (hydroponic conditions), the shoots of plants were harvested, dried in an air incubator at $60^{\circ} \mathrm{C}$ for more than $60 \mathrm{hr}$, and the dry weights were measured. The tissues were digested with $3 \mathrm{ml}$ of $61 \% \mathrm{HNO}_{3}$ (for boron determination; Wako Pure Chemicals, Osaka, Japan) in a tube at $110^{\circ} \mathrm{C}$ in a DigiPREP apparatus (SCP Science, Quebec, Canada) until complete dryness. The residues were dissolved in $10 \mathrm{ml}$ of $2 \% \mathrm{HNO}_{3}$ and analyzed for elements by inductively coupled plasma mass spectrometry (ICP-MS) (ELAN, DRC-e; Perkin-Elmer, Waltham, MA, USA).

For Aberdeen assays, the leaves were cleaned by rinsing with ultrapure water and placed into Pyrex digestion tubes. Samples were dried in an oven at $88^{\circ} \mathrm{C}$ for $20 \mathrm{hr}$. After cooling, seven reference samples from each planted block were weighed. The samples together with blank controls were digested with $0.90 \mathrm{ml}$ concentrated nitric acid (Baker Instra-Analyzed; Avantor Performance Materials) and diluted to $10.0 \mathrm{ml}$ with ultrapure water $(18.2 \mathrm{M} \Omega \mathrm{cm})$. The internal standard Indium (In) was added to the acid prior to digestion for monitoring technical errors and plasma stability in the ICP-MS instrument. After samples and controls were prepared, elemental analysis was performed with an ICP-MS (NexION 300D; PerkinElmer) coupled to Apex desolvation system and SC-4 DX autosampler (Elemental Scientific Inc., Omaha, NE, USA), monitoring these elements: B, Na, Mg, P, K, Ca, Mn, Fe, $\mathrm{Co}, \mathrm{Cu}, \mathrm{Zn}$, $\mathrm{Sr}$, and Mo. All samples were normalized to calculated weights, as determined with a heuristic algorithm using the best-measured elements, the weights of the seven weighed samples and the solution concentrations (Lahner et al., 2003), detailed at www.ionomicshub.org.

For ionic chromatography, for each sample one rosette leaf of a 5-week-old plant was harvested, put in $30 \mathrm{ml}$ Nanopure water and incubated at $80^{\circ} \mathrm{C}$ for $1 \mathrm{hr}$. Each sample was then filtered with a $0.45-\mu \mathrm{m}$ non-pyrogenic sterile filter (Sarstedt, Germany). The samples were then diluted to reach a final concentration of $0.83 \mathrm{mg}$ fresh weight per $1 \mathrm{ml}$. lon concentrations were obtained with an ion chromatography system (ICS-1100/2100, Dionex-Thermo Fischer).

\section{Expression analysis}

Microarray analysis

WT and sgn3-3 plants were grown in hydroponic conditions and material was harvested 24 days post-stratification. Roots and rosettes were collected separately, and each sample is a mix of three 
plants. Each type of sample was harvested in triplicate. In these growth conditions, sgn3-3 was nearly indistinguishable from wild type. Total RNAs were isolated and purified with RNeasy Plant Mini Kit (Qiagen, Venlo, The Netherlands). RNA quantities were assessed with a NanoDropND-1000 spectrophotometer and RNA qualities with an RNA 6000 NanoChips with the Agilent 2100 Bioanalyzer (Agilent, Palo Alto, USA). For each sample, $300 \mathrm{ng}$ of total RNA were amplified using the message amp II enhanced (AM1791, Ambion) kit. $12.5 \mu \mathrm{g}$ of the resulting biotin-labeled cRNA was chemically fragmented. Affymetrix ATH1 arrays (Affymetrix, Santa Clara, CA) were hybridized with $11 \mu \mathrm{g}$ of fragmented target at $45^{\circ} \mathrm{C}$ for $17 \mathrm{hr}$ washed and stained according to the protocol described in Affymetrix GeneChip Expression Analysis Manual (Fluidics protocol FS450_0007). The arrays were scanned using the GeneChip Scanner 30007 G (Affymetrix). All statistical analyses were performed using the free high-level interpreted statistical language $\mathrm{R}$ and various Bioconductor packages (http://www. Bioconductor.org). Hybridization quality was assessed using the Expression Console software (Affymetrix). Normalized expression signals were calculated from Affymetrix CEL files using RMA normalization method. Differential hybridized features were identified using Bioconductor package 'limma' that implements linear models for microarray data (Smyth, 2004). The p-values were adjusted for multiple testing with Benjamini and Hochberg's method to control the false discovery rate (FDR) (Benjamini and Hochberg, 1995). Genevestigator was used to analyze the effect of nutritional stress on the expression of genes deregulated in sgn3 (Hruz et alo, 2008).

\section{Real time Q-PCR}

Total RNA was extracted using TRIzol reagent (Invitrogen) and purified using RNeasy MinElute Cleanup Kit (Qiagen) after DNase treatment (Qiagen). The integrity of DNA-free RNA was verified by PCR, agarose gel electrophresis, and nanodrop.

An equal amount of total RNA ( $3 \mu \mathrm{g}$ ) was used for RT with anchored oligo(dT23). Real time Q-PCR was performed using a Stratagene MxPro 3005P Real-Time PCR System (Stratagene, La Jolla, CA). Three biological replicates performed in three technical replicates were analyzed for each sample. The specificity of each amplification product was verified by DNA melting curve analysis and gel electrophoresis of the amplified products. Relative transcript levels $(\mathrm{RTL})$ were calculated relative to the transcript level of the reference gene EF1 $\alpha$, as follows: RTL $=1000 \times 2^{-\Delta C t}$ with $\Delta C t$, the change in cycle threshold between the target gene and the reference gene. The following oligonucleotides were used EF1a (At1g07920, At1g07930, At1g07940) 5'-GTCGATTCTGGAAAGTCGACC-3' and 5'-AATGTCAATGG TGATACCACGC-3'; CASP1 (At2g36100) 5'-AGAGAGGTTTGGCTATATT-3' and 5'-CTACGGCTATCA CAAAGTA-3'; CASP4 (At5g06200) 5'-AGACTTCTCTTGCTTGTTCT-3' and 5'-GACAGAAATCTCC AAACTG-3'; AKT1 (At2G26650) 5'-TCTAAATTGTGTTCTTCTTCTGTTGGA-3' and 5'-CCTTCCGCG TCTCTGCAA-3'; HAK5 (At4g13420) 5'-CGAGACGGACAAAGAAGAGGAACC-3' and 5'- CACGA CCCTTCCCGACCTAATCT-3'.

\section{Statistical analysis}

All statistical analyses were done in the R environment (http://www.R-project.org/). Binary comparisons between wild type and sgn3 were performed using Student's t-test. Multiple comparisons between wild type, sgn3-3, and sgn3-4 mutants, one-way ANOVA was performed, and Tukey's test was subsequently used as a multiple comparison procedure.

\section{SGN3 domain identification}

The different features were predicted using the following web resources. Signal peptide, Leucine-rich repeats, transmembrane domain, and kinase domain: http://www.uniprot.org/uniprot/COLGQ5; LRR $\mathrm{N}$-terminal domain: http://pfam.sanger.ac.uk/search/sequence; kinase sub-domains were defined with reference to Hanks and Hunter (1995) and by aligning to the BRI1 kinase domain (Vert et al., 2005). Borders between kinase sub-domains were set manually by comparison of amino acids in the respective border regions for PKA C alpha and BRI1. In case of conflict, the BRI1 annotation was considered as more meaningful because of its plant origin.

\section{Acknowledgements}

We thank the Genomic Technologies Facility (GTF) and the Central Imaging Facility (CIF) of the University of Lausanne for expert technical support. We thank Valérie Dénervaud Tendon, Guillaume Germion, Deborah Mühlemann, and Kayo Konishi for technical assistance and John Danku and Véronique Vacchina for ICP-MS analysis. This work was funded by grants from the Swiss National 
Science Foundation (SNSF), the European Research Council (ERC) to NG and a Human Frontiers Science Program (HFSP) grant to JT and NG. GL and CM were supported by the Agropolis foundation (Rhizopolis) and the Agence Nationale de la Recherche (HydroRoot; ANR-11-BSV6-018). MB was supported by a EMBO long-term postdoctoral fellowship, JEMV by a Marie Curie IEF fellowship and TK by the Japan Society for the Promotion of Sciences (JSPS).

\section{Additional information}

\begin{tabular}{ll}
$\begin{array}{l}\text { Funding } \\
\text { Funder }\end{array}$ & Author \\
\hline European Research Council & Alexandre Pfister, \\
& Marie Barberon, \\
& Julien Alassimone, \\
& Lothar Kalmbach, \\
& Yuree Lee \\
\hline Swiss National Science Foundation & Alexandre Pfister, \\
& Marie Barberon, \\
& Julien Alassimone, \\
& Lothar Kalmbach, \\
& Yuree Lee \\
\hline
\end{tabular}

The funders had no role in study design, data collection and interpretation, or the decision to submit the work for publication.

Author contributions

AP, MB, JA, LK, YL, JEMV, Conception and design, Acquisition of data, Analysis and interpretation of data, Drafting or revising the article; MY, Acquisition of data, Contributed unpublished essential data or reagents; GL, Acquisition of data, Analysis and interpretation of data; CM, JT, DES, NG, Conception and design, Analysis and interpretation of data, Drafting or revising the article; TK, DR, Conception and design, Acquisition of data, Analysis and interpretation of data

\section{References}

Alassimone J, Naseer S, Geldner N. 2010. A developmental framework for endodermal differentiation and polarity. Proceedings of the National Academy of Sciences of USA 107:5214-5219. doi: 10.1073/pnas. 0910772107.

Balda MS, Matter K. 2009. Tight junctions and the regulation of gene expression. Biochimica et Biophysica Acta 1788:761-767. doi: 10.1016/j.bbamem.2008.11.024.

Baxter IR, Vitek O, Lahner B, Muthukumar B, Borghi M, Morrissey J, Guerinot ML, Salt DE. 2008. The leaf ionome as a multivariable system to detect a plant's physiological status. Proceedings of the National Academy of Sciences of USA 105:12081-12086. doi: 10.1073/pnas.0804175105.

Behrisch R. 1926. Zur Kenntnis der Endodermiszelle. Berichte der Deutschen Botanischen Gesellschaft 44:162-164.

Benjamini Y, Hochberg Y. 1995. Controlling the false discovery rate - a practical and powerful approach to multiple testing. Journal of the Royal Statistical Society Series B-Methodological 57:289-300.

Birnbaum K, Shasha DE, Wang JY, Jung JW, Lambert GM, Galbraith DW, Benfey PN. 2003. A gene expression map of the Arabidopsis root. Science 302:1956-1960. doi: 10.1126/science.1090022.

Brady SM, Orlando DA, Lee JY, Wang JY, Koch J, Dinneny JR, Mace D, Ohler U, Benfey PN. 2007. A high-resolution root spatiotemporal map reveals dominant expression patterns. Science 318:801-806. doi: 10.1126/ science. 1146265.

Cheung AY, Wu HM. 2011. THESEUS 1, FERONIA and relatives: a family of cell wall-sensing receptor kinases? Current Opinion in Plant Biology 14:632-641. doi: 10.1016/j.pbi.2011.09.001.

Clarkson DT. 1984. Calcium transport between tissues and its distribution in the plant. Plant, Cell \& Environment 7:449-456. doi: 10.1111/j.1365-3040.1984.tb01435.x.

Clough SJ, Bent AF. 1998. Floral dip: a simplified method for Agrobacterium-mediated transformation of Arabidopsis thaliana. The Plant Journal 16:735-743. doi: 10.1046/j.1365-313x.1998.00343.x.

Fujiwara T, Hirai MY, Chino M, Komeda Y, Naito S. 1992. Effects of sulfur nutrition on expression of the soybean seed storage protein genes in transgenic petunia. Plant Physiology 99:263-268. doi: 10.1104/ pp.99.1.263.

Geldner N. 2009. Cell polarity in plants: a PARspective on PINs. Current Opinion in Plant Biology 12:42-48. doi: $10.1016 /$ j.pbi.2008.09.009. 
Geldner N. 2013. The endodermis. Annual Review of Plant Biology 64:531-558. doi: 10.1146/annurev-arplant050312-120050.

Hanks SK, Hunter T. 1995. Protein kinases 6. The eukaryotic protein kinase superfamily: kinase (catalytic) domain structure and classification. FASEB Journal 9:576-596.

Hosmani PS, Kamiya T, Danku J, Naseer S, Geldner N, Guerinot ML, Salt DE. 2013. Dirigent domain-containing protein is part of the machinery required for formation of the lignin-based Casparian strip in the root. Proceedings of the National Academy of Sciences of USA 110:14498-14503. doi: 10.1073/pnas.1308412110.

Hruz T, Laule O, Szabo G, Wessendorp F, Bleuler S, Oertle L, Widmayer P, Gruissem W, Zimmermann P. 2008. Genevestigator v3: a reference expression database for the meta-analysis of transcriptomes. Advances in Bioinformatics 2008:420747. doi: 10.1155/2008/420747.

Javot H, Lauvergeat V, Santoni V, Martin-Laurent F, Guclu J, Vinh J, Heyes J, Franck KI, Schaffner AR, Bouchez D, Maurel C. 2003. Role of a single aquaporin isoform in root water uptake. The Plant Cell 15:509-522. doi: 10.1105/ tpc.008888.

Krömer K. 1903. Wurzelhaut, Hypodermis und Endodermis der Angiospermenwurzel. Bibliotheca Botanica 59:156.

Lahner B, Gong J, Mahmoudian M, Smith EL, Abid KB, Rogers EE, Guerinot ML, Harper JF, Ward JM, Mcintyre L, Schroeder JI, Salt DE. 2003. Genomic scale profiling of nutrient and trace elements in Arabidopsis thaliana. Nature Biotechnology 21:1215-1221. doi: 10.1038/nbt865.

Lee Y, Rubio MC, Alassimone J, Geldner N. 2013. A mechanism for localized lignin deposition in the endodermis. Cell 153:402-412. doi: 10.1016/j.cell.2013.02.045.

Lindner H, Muller LM, Boisson-Dernier A, Grossniklaus U. 2012. CrRLK1L receptor-like kinases: not just another brick in the wall. Current Opinion in Plant Biology 15:659-669. doi: 10.1016/j.pbi.2012.07.003.

Marschner H, Marschner P. 2012. Marschner's mineral nutrition of higher plants. London; Waltham, MA: Elsevier/ Academic Press.

Naseer S, Lee Y, Lapierre C, Franke R, Nawrath C, Geldner N. 2012. Casparian strip diffusion barrier in Arabidopsis is made of a lignin polymer without suberin. Proceedings of the National Academy of Sciences of USA 109:10101-10106. doi: 10.1073/pnas.1205726109.

Oda Y, Fukuda H. 2012. Initiation of cell wall pattern by a Rho- and microtubule-driven symmetry breaking Science 337:1333-1336. doi: 10.1126/science.1222597.

Postaire O, Tournaire-Roux C, Grondin A, Boursiac Y, Morillon R, Schaffner AR, Maurel C. 2010. A PIP1 aquaporin contributes to hydrostatic pressure-induced water transport in both the root and rosette of Arabidopsis. Plant Physiology 152:1418-1430. doi: 10.1104/pp.109.145326.

Racolta A, Bryan AC, Tax FE. 2014. The receptor-like kinases GSO1 and GSO2 together regulate root growth in Arabidopsis through control of cell division and cell fate specification. Developmental Dynamics 243:257-278. doi: 10.1002/dvdy.24066.

Roppolo D, De Rybel B, Tendon VD, Pfister A, Alassimone J, Vermeer JE, Yamazaki M, Stierhof YD, Beeckman T, Geldner N. 2011. A novel protein family mediates Casparian strip formation in the endodermis. Nature 473:380-383. doi: 10.1038/nature10070.

Roppolo D, Geldner N. 2012. Membrane and walls: who is master, who is servant? Current Opinion in Plant Biology 15:608-617. doi: 10.1016/j.pbi.2012.09.009.

Smyth GK. 2004. Linear models and empirical bayes methods for assessing differential expression in microarray experiments. Statistical Applications in Genetics and Molecular Biology 3:Article3. doi: 10.2202/15446115.1027.

Takano J, Yamagami M, Noguchi K, Hayashi H, Fujiwara T. 2001. Preferential translocation of boron to young leaves in Arabidopsis thaliana regulated by the BOR1 gene. Soil Science and Plant Nutrition 47:345-357. doi: 10.1080/00380768.2001.10408398.

Thibault JF, Rinaudo M. 1985. Interactions of mono-valent and divalent counterions with alkali-deesterified and enzyme-deesterified pectins in salt-free solutions. Biopolymers 24:2131-2143. doi: 10.1002/bip. 360241109.

Tsuwamoto R, Fukuoka H, Takahata Y. 2008. GASSHO1 and GASSHO2 encoding a putative leucine-rich repeat transmembrane-type receptor kinase are essential for the normal development of the epidermal surface in Arabidopsis embryos. The Plant Journal 54:30-42. doi: 10.1111/j.1365-313X.2007.03395.x.

Vert G, Nemhauser JL, Geldner N, Hong F, Chory J. 2005. Molecular mechanisms of steroid hormone signaling in plants. Annual Review of Cell and Developmental Biology 21:177-201. doi: 10.1146/annurev.cellbio. 21.090704.151241.

Waisel Y, Eshel A, Kafkafi U. 2002. Plant roots: the hidden half. New York: M. Dekker.

Wang Y, Wu WH. 2013. Potassium transport and signaling in higher plants. Annual Review of Plant Biology 64:451-476. doi: 10.1146/annurev-arplant-050312-120153.

Wegner LH. 2014. Root pressure and beyond: energetically uphill water transport into xylem vessels? Journal of Experimental Botany 65:381-393. doi: 10.1093/jxb/ert391.

White PJ. 2001. The pathways of calcium movement to the xylem. Journal of Experimental Botany 52:891-899. doi: 10.1093/jexbot/52.358.891.

White PJ, Broadley MR. 2003. Calcium in plants. Annals of Botany 92:487-511. doi: 10.1093/aob/mcg164.

Xing Q, Creff A, Waters A, Tanaka H, Goodrich J, Ingram GC. 2013. ZHOUPI controls embryonic cuticle formation via a signalling pathway involving the subtilisin protease ABNORMAL LEAF-SHAPE1 and the receptor kinases GASSHO1 and GASSHO2. Development 140:770-779. doi: 10.1242/dev.088898. 